\title{
A More Unified Approach to Free Logics
}

\section{Edi Pavlović ${ }^{1}$ (1) · Norbert Gratzl $\left.\right|^{2}$ (1)}

Received: 13 December 2019 / Accepted: 23 June 2020 / Published online: 17 August 2020

(C) The Author(s) 2020

\begin{abstract}
Free logics is a family of first-order logics which came about as a result of examining the existence assumptions of classical logic. What those assumptions are varies, but the central ones are that (i) the domain of interpretation is not empty, (ii) every name denotes exactly one object in the domain and (iii) the quantifiers have existential import. Free logics usually reject the claim that names need to denote in (ii), and of the systems considered in this paper, the positive free logic concedes that some atomic formulas containing non-denoting names (namely self-identity) are true, while negative free logic rejects even the latter claim. Inclusive logics, which reject (i), are likewise considered. These logics have complex and varied axiomatizations and semantics, and the goal of this paper is to present an orderly examination of the various systems and their mutual relations. This is done by first offering a formalization, using sequent calculi which possess all the desired structural properties of a good proof system, including admissibility of contraction and cut, while streamlining free logics in a way no other approach has. We then present a simple and unified system of abstract semantics, which allows for a straightforward demonstration of the meta-theoretical properties, and offers insights into the relationship between different logics (free and classical). The final part of this paper is dedicated to extending the system with modalities by using a labeled sequent calculus, and here we are again able to map out the different approaches and their mutual relations using the same framework.
\end{abstract}

Keywords Positive free logic · Negative free logic $\cdot$ Sequent calculus · G3 ·

Modal logic

\footnotetext{
An anonymous reviewer has offered numerous suggestions which have, combined, amounted to a significant improvement of the paper, and for that we extend our gratitude. Special thanks goes to O. Foisch.
}

Edi Pavlović

Edi.Pavlovic@helsinki.fi

Extended author information available on the last page of the article. 


\section{Introduction}

This is a paper in proof theory, specifically in sequent calculi. It has three main goals - first to present a unified approach to the proof theory of free logics (with one caveat to be mentioned shortly), second, to offer a unified, streamlined and above all fruitful semantical approach to those free logics, and finally to extend the results of the first two goals to applications to both inclusive and non-inclusive systems, and all of those to modal logics.

Before we delve deeper let us briefly remind ourselves what a free logic is standardly understood to be. Free logics is a family of first-order logics which came about as a result of examining the existence assumptions of classical logic [8,9]. The term is due to Karel Lambert [9-11] and is short for first order logic free of existential assumptions, and we take a logic to be free iff (1) it is free of existential presuppositions with respect to its singular terms, (2) it is free of existential presuppositions with respect to its general terms and finally (3) its quantifiers have existential import (the last claim is expressed by the axioms A3 and A4 later in the paper) [6]. The three (families of) free logics we will here distinguish depend on their decisions on the truth values of (atomic) statements containing empty terms. If a free logic holds that those can be true it is called positive, if it holds that they are false it is called negative and if it holds they take a third value it is called neutral.

In the light of recent research on structural proof theory it seems timely to have a fresh look at the family of free logics. Our focus is on positive and negative free logics (according to our definitions above) and not on neutral free logics. Herein lies the caveat mentioned in the beginning. Neutral free logics have been studied (see [12]) but up to now still lack the rigorous systematicity the other two family members enjoy. For us the main reasons for this is that there is some lack of formal intuitions or even a clash of formal intuitions when it comes to, say, the relationship between the semantics stati of sentences/formulas with empty singular terms in the contexts of the existence predicate, and identity statements as well as to a philosophically satisfying account of the conditional in this context.

For our proof-theoretic investigations we have chosen the popular G3-style systems. This makes the presentations of the formal systems rather concise and dispenses with structural rules at the core of the systems altogether. Furthermore it has the advantage of putting the insights from $[18,19]$ on the identity predicate to full use.

The approach to semantics we utilize in this paper - which we label generalized semantics - is a purely syntactic representation of semantics, with the main goal being to simplify comparisons between logical systems. Given how predictable (cutfree) derivations are in a G3 systems, the comparison can be achieved simply by noting that the semantics of one system require comparatively higher restrictions (and those are captured in a systematic way in a sequent calculus). So we are able to focus on the presence and absence of individual semantic restrictions. These in turn allow for descriptions of different systems in terms of a series of binary choices, and thus significantly streamline the way in which they can be systematized. In a nutshell, this allows for a comparisons between whole systems in a point-by-point manner. 
Plan of the paper: in the following section we present a G3-style sequent calculus for both positive and negative free logic, and then demonstrate a range of desirable structural properties, including being cut- and contraction free. We establish the connection to previous sequent calculi for free logics and moreover we also show that our formalization of positive and negative free logic is adequate by showing that all the axioms of their standard representations are derivable. In Section 3 we present a generalized version of the semantics for positive and negative free logic, which allows for a systematic representation of a wide range of logics and their relations, primarily positive and negative free logic, as well as classical logic, on both inclusive and non-inclusive versions. Moreover, this generalized semantics has further uses in the discussion of modal logic. That, however, is left for a later section and we first utilize the general semantics to prove the soundness and completeness of our calculi in Section 4. After a discussion of some further logics captured by our systematization in Section 5, in Section 6 we examine the modal extension of both the calculi and the generalized semantics, again show they possess the structural and metatheoretic properties, and discuss the different approaches to identity that our formalization allows for. Finally in Section 7 we look at further avenues of research that this paper opens. The appendices contain some useful technical results that have been omitted from the main body of the paper for conciseness.

\section{The Formal Systems $\mathbf{G 3}_{\mathrm{pf}}$ and $\mathbf{G}_{\mathrm{nf}}$}

In formulating the rules for the free logics discussed here we take as our starting point the sequent calculus for the negative variety first introduced in [6]. We then streamline it by transforming it into a G3-style calculus along the lines of [19], and to a lesser extent, $[16,20]$. Specifically, we replace multiple types of initial sequents with only a single one, and we simplify the quantifier rules. We defer showing that the new versions of the rules are adequate and deductively equivalent to the previous ones, as well as their comparative advantages, for after the discussion of the structural properties of the new systems.

\subsection{Language of Free Logics}

The language $\mathcal{L}$ utilized in this paper is a standard first order language without functions, adapted from [6], with the vocabulary defined as

Definition 2.1 (Alphabet $\mathcal{L}$ ) The alphabet of the language $\mathcal{L}$ consists of:

1. Denumerable list of free individual variables (names): $a, b, c, \ldots$,

2. Denumerable list of bound individual variables: $x, y, z, \ldots$,

3. Denumerable list of $n$-ary predicate variables, including a unary predicate $E$ ! and a binary predicate $=$,

4. ᄀ, $\wedge, \vee, \rightarrow, \forall, \exists,($,$) .$ 
The metalinguistic variables we use in this paper are as follows:

Definition 2.2 (Metalinguistic variables) 1. Free individual variables: $t, s, d$,

2. Bound individual variables: $x, y, z$,

3. Terms (free or bound individual variables): $\bar{t}, \bar{s}$,

4. $n$-ary predicates: $P^{n}$,

5. Atoms: $P$,

6. Formulas: $A, B$, where $A[\bar{t}]$ denotes formula $A$ containing the term $\bar{t}, A[\bar{t} / / \bar{s}]$ the result of substituting (any number of) $\bar{s}$ by $\bar{t}$ in $A, A[\bar{t} / \bar{s}]$ the result of substituting every $\bar{s}$ by $\bar{t}$ in $A$, and $A[t / x]$ the result of a substitution of all the free instances of $x$ by $t$ in $A$.

A formula of our language $\mathcal{L}$ is defined as

Definition 2.3 (Formula of $\mathcal{L}$ )

$$
A::=P^{n}\left(\overline{t_{1}}, \ldots, \overline{t_{n}}\right)|\neg A| A \circ A|\forall x A| \exists x A
$$

where $\circ \in\{\wedge, \vee, \rightarrow\}$.

In the rest of the paper, unless otherwise specified we use variables to refer to bound individual variables, names to refer to free individual variables, and formulas to refer to closed formulas (i.e. formulas in which every free occurrence of a term is a name).

\subsection{Positive Free Logic G3 pf}

The base from which we will develop the system of positive free logic $G 3_{p f}$ (and shortly also the negative version) is quantified classical calculus $G 3_{c}$ [19] in Fig. 1. The basic unit of a sequent calculus is a sequent $\Gamma \Rightarrow \Delta$, where $\Gamma$ and $\Delta$ are finite multisets of (closed) formulas. All the formulas except $\Gamma$ and $\Delta$ are called active formulas of the rule if they occur only in the upper sequent(s) and principal if they occur in the lower sequent of the rule.

The positive free logic $G 3_{p f}$ (Fig. 2) is then obtained by replacing the quantifier rules in $G 3_{c}$ with the rules below, i.e. by adding the condition that $E ! t$. This approach from $[16,20]$ was explicitly used in [14] to simplify the quantifier rules of intuitionistic logic with existence predicate. However, in the more general framework of this paper, $E$ ! is for left undefined and open to multiple interpretations (cf. [1, 4]).

\subsection{Negative Free Logic G3 nf}

The negative free logic $G 3_{n f}$ (Fig. 3) is obtained by replacing the rule $={ }_{R e f}$ (reflexivity of identity) in $G 3_{p f}$ by the rules:

We can demonstrate a few simple facts about these systems. Full proofs can be found in Appendix A, and here we just note that 
Initial sequent: $P, \Gamma \Rightarrow \Delta, P$

\section{Propositional rules:}

$$
\begin{array}{ll}
\left.\frac{\Gamma \Rightarrow \Delta, A}{\neg A, \Gamma \Rightarrow \Delta} \mathrm{L}\right\urcorner & \left.\frac{A, \Gamma \Rightarrow \Delta}{\Gamma \Rightarrow \Delta, \neg A} \mathrm{R}\right\urcorner \\
\frac{A, B, \Gamma \Rightarrow \Delta}{A \wedge B, \Gamma \Rightarrow \Delta} \mathrm{L} \wedge & \frac{\Gamma \Rightarrow \Delta, A \quad \Gamma \Rightarrow \Delta, B}{\Gamma \Rightarrow \Delta, A \wedge B} \mathrm{R} \wedge \\
\frac{A, \Gamma \Rightarrow \Delta \quad B, \Gamma \Rightarrow \Delta}{A \vee B, \Gamma \Rightarrow \Delta} \mathrm{L} \vee & \frac{\Gamma \Rightarrow \Delta, A, B}{\Gamma \Rightarrow \Delta, A \vee B} \mathrm{R} \vee \\
\frac{\Gamma \Rightarrow \Delta, A \quad B, \Gamma \Rightarrow \Delta}{A \rightarrow B, \Gamma \Rightarrow \Delta} \mathrm{L} \rightarrow & \frac{A, \Gamma \Rightarrow \Delta, B}{\Gamma \Rightarrow \Delta, A \rightarrow B} \mathrm{R} \rightarrow
\end{array}
$$

Identity rules:

$$
\frac{t=t, \Gamma \Rightarrow \Delta}{\Gamma \Rightarrow \Delta}={ }_{R e f} \quad \frac{s=t, P[s / / t], P[t], \Gamma \Rightarrow \Delta}{s=t, P[t], \Gamma \Rightarrow \Delta}={ }_{R e p l}
$$

\section{Quantifier rules:}

$$
\begin{array}{ll}
\frac{A[t / x], \forall x A, \Gamma \Rightarrow \Delta}{\forall x A, \Gamma \Rightarrow \Delta} \mathrm{L} \forall & \frac{\Gamma \Rightarrow \Delta, A[t / x]}{\Gamma \Rightarrow \Delta, \forall x A} \mathrm{R} \forall^{*} \\
\frac{A[t / x], \Gamma \Rightarrow \Delta}{\exists x A, \Gamma \Rightarrow \Delta} \mathrm{L} \exists^{*} & \frac{\Gamma \Rightarrow \Delta, \exists x A, A[t / x]}{\Gamma \Rightarrow \Delta, \exists x A} \mathrm{R} \exists
\end{array}
$$

Where $P$ is atomic, and $t$ is fresh in rules marked with *

Fig. $1 \mathrm{G} 3_{c}$

\section{Quantifier rules:}

$$
\begin{aligned}
& \frac{A[t / x], E ! t, \forall x A, \Gamma \Rightarrow \Delta}{E ! t, \forall x A, \Gamma \Rightarrow \Delta} \mathrm{L} \forall \quad \frac{E ! t, \Gamma \Rightarrow \Delta, A[t / x]}{\Gamma \Rightarrow \Delta, \forall x A} \mathrm{R} \forall^{*} \\
& \frac{E ! t, A[t / x], \Gamma \Rightarrow \Delta}{\exists x A, \Gamma \Rightarrow \Delta} \mathrm{L} \exists * \quad \frac{E ! t, \Gamma \Rightarrow \Delta, \exists x A, A[t / x]}{E ! t, \Gamma \Rightarrow \Delta, \exists x A} \mathrm{R} \exists
\end{aligned}
$$

* $t$ fresh.

Fig. 2 G $3_{p f}$

$$
\frac{E ! t, P[t], \Gamma \Rightarrow \Delta}{P[t], \Gamma \Rightarrow \Delta} E !^{*} \quad \frac{t=t, P[t], \Gamma \Rightarrow \Delta}{P[t], \Gamma \Rightarrow \Delta}={ }_{R e f} *
$$

* $P$ atomic.

Fig. $3 \mathrm{G} 3_{n f}$ 
Lemma 2.4 ( $\alpha$-converison) A derivation (in either $G 3_{p f}$ or $G 3_{n f}$ ), where $\vdash_{n}$ denotes derivability with height bounded by $n$, of $\vdash_{n} \Gamma \Rightarrow \Delta$ can be converted into a derivation of $\vdash_{n} \Gamma^{\prime} \Rightarrow \Delta^{\prime}$, where $\Gamma^{\prime}$ and $\Delta^{\prime}$ differ from $\Gamma$ and $\Delta$, respectively, only in namings of bound variables.

Note that this result justifies the decision to keep the free and bound individual variables separate.

Lemma 2.5 (Substitution) If $\vdash_{n} \Gamma \Rightarrow \Delta$ is derivable in $G 3_{p f}\left(G 3_{n f}\right)$, then $\vdash_{n}$ $\Gamma[t / s] \Rightarrow \Delta[t / s]$ is derivable.

\subsection{Structural Properties}

The systems $G 3_{p f}$ and $G 3_{n f}$ possess a range of desirable proof-theoretic properties. Again these proofs are left for Appendix A, where important parts are shown. Here we simply state that

Lemma 2.6 (Axiom generalization) Any sequent of the form $A, \Gamma \Rightarrow \Delta, A$ is derivable in $G 3_{p f}$ and $G 3_{n f}$.

Lemma 2.7 (Weakening) Weakening is height-preserving admissible in G3 $3_{p f}$ and $G 3_{n f}$ :

i) If $\vdash_{n} \Gamma \Rightarrow \Delta$ then $\vdash_{n} C, \Gamma \Rightarrow \Delta$.

ii) If $\vdash_{n} \Gamma \Rightarrow \Delta$ then $\vdash_{n} \Gamma \Rightarrow \Delta, C$.

Lemma 2.8 (Invertibility) All the rules of $G 3_{p f}$ and $G 3_{n f}$ are height-preserving invertible.

Lemma 2.9 (Contraction) Contraction is height-preserving admissible in G3 $3_{p f}$ and $G 3_{n f}$ :

i) If $\vdash_{n} C, C, \Gamma \Rightarrow \Delta$ then $\vdash_{n} C, \Gamma \Rightarrow \Delta$.

ii) If $\vdash_{n} \Gamma \Rightarrow \Delta, C, C$ then $\vdash_{n} \Gamma \Rightarrow \Delta, C$.

Theorem 2.10 Cut is admissible in $G 3_{p f}$ and $G 3_{n f}$.

The proofs of all of these become routine, following a general pattern of demonstration from [19], once appropriate modifications are made to account for the specificities of the system discussed here.

From the last theorem it immediately follows that

Corollary 2.11 (Weak subformula property) Every formula occurring in a derivation of $\Gamma \Rightarrow \Delta$ is either a subformula (under standard definition) of some formula occurring in $\Gamma, \Delta$, or atomic.

and from there 
Corollary 2.12 (Consistency) The calculi $G 3_{p f}$ and $G 3_{n f}$ are consistent.

\subsection{Relation to Previous Systems of Free Logic}

The sequent calculi for free logics offered so far, e.g. for either version of free logics in [2] (here simplified to be context-sharing) or specifically for negative free logic in [6] use the following versions of the quantifier rules for $\mathrm{L} \forall$ and $\mathrm{R} \exists$ :

$$
\begin{aligned}
& \frac{\forall x A, \Gamma \Rightarrow \Delta, E ! t \quad A[t / x], \forall x A, \Gamma \Rightarrow \Delta}{\forall x A, \Gamma \Rightarrow \Delta} \mathrm{L}^{\dagger} \\
& \qquad \frac{\Gamma \Rightarrow \Delta, \exists x A, E ! t \quad \Gamma \Rightarrow \Delta, \exists x A, A[t / x]}{\Gamma \Rightarrow \Delta, \exists x A} \mathrm{R}^{\dagger}
\end{aligned}
$$

Notably, these are both two-premise rules, whereas the rules we use are onepremise. The approaches are deductively equivalent, but ours has multiple advantages. We first demonstrate the former claim:

Lemma 2.13 The rules $L \forall^{\dagger}$ and $R \exists^{\dagger}$ are admissible in $G 3_{p f}$ and $G 3_{n f}$.

Proof We illustrate on the example of $\mathrm{L}^{\dagger}$, with the other being nearly identical, and make use of the structural properties of $\mathrm{G} 3{ }_{p f}$ and $\mathrm{G} 3_{n f}$.

$$
\begin{gathered}
\frac{A[t / x], \forall x A, \Gamma \Rightarrow \Delta}{E ! t, A[t / x], \forall x A, \Gamma \Rightarrow \Delta} \text { Lemma } 2.7 \\
\forall x A, \Gamma \Rightarrow \Delta, E ! t \quad \frac{E ! t, \forall x A, \Gamma \Rightarrow \Delta}{\forall} \text { Theorem 2.10, Lemma } 2.9
\end{gathered}
$$

Lemma 2.14 The quantifier rules of $G 3_{p f}$ and $G 3_{n f}$ are admissible in a system with $L \forall^{\dagger}$ and $R \exists^{\dagger}$.

Proof We again illustrate on the example of $\mathrm{L} \forall$. Note that the upper left sequent is initial.

$$
\frac{E ! t, \forall x A, \Gamma \Rightarrow \Delta, E ! t \quad A[t / x], E ! t, \forall x A, \Gamma \Rightarrow \Delta}{E ! t, \forall x A, \Gamma \Rightarrow \Delta} \mathrm{L}^{\dagger}
$$

The case for the rules $E !$ and $={ }_{\text {Ref }}$ is straightforward as they replace initial sequents.

\subsubsection{Advantages of the Present Approach}

The first advantage of our approach is that it simplifies both proof-search and metatheory by virtue of not creating a branching when the rules are read bottom-up. The second is that in this formulation, the rules exhibit a perfect symmetry with the modal operator rules in their typical formulation, as in [16, 20]. Finally, the rules do not tacitly rely on an implicit conditional and conjunction introduction, respectively for $\mathrm{L} \forall$ and $\mathrm{R} \exists$, as had been the case with previous iterations. For example, this was the 
case with the free logic rules in [24, pp. 200-201], where the definitions make this reliance explicit, while here the quantifiers are restored to being independent of other operators (see also [22]).

\subsubsection{Adequacy of the Systems $G 3_{p f}$ and $G 3_{n f}$}

In this section we demonstrate the adequacy of the formalization in the systems $G 3_{p f}$ and $G 3_{n f}$ by showing that each proves (one version of) an axiomatization [15, 21] of their respective system. In addition to the admissibility of modus ponens, this also serves as an indirect proof of completeness of the systems, but we will not focus on that as a direct proof of it will be presented in a later section.

An axiomatization of positive free logic is the following.

Definition 2.15 (Positive free logic, PFL)

1. $\forall x(A \rightarrow B) \rightarrow(\forall x A \rightarrow \forall x B)$

2. $A \rightarrow \forall x A(x$ not free in $A)$

3. $\forall x A \rightarrow(E ! t \rightarrow A[t / x])$

4. $\forall x E ! x$

5. $s=t \rightarrow(A \rightarrow A[t / / s])$

6. $t=t$

An axiomatization of negative free logic is:

Definition 2.16 (Negative free logic, NFL)

1. $\forall x(A \rightarrow B) \rightarrow(\forall x A \rightarrow \forall x B)$

2. $A \rightarrow \forall x A(x$ not free in $A)$

3. $\forall x A \rightarrow(E ! t \rightarrow A[t / x])$

4. $\forall x E ! x$

5. $s=t \rightarrow(A \rightarrow A[t / / s])$

6. $\forall x(x=x)$

7. $P t_{1}, \ldots, t_{n} \rightarrow E ! t_{i}, 1 \leq i \leq n$

In either system the only rule of inference is modus ponens.

We first proceed to establish two auxiliary results, namely the admissibility of modus ponens and generalization of $={ }_{\text {Repl }}$. The former is simple but it will help with the legibility of the proofs later on.

Lemma 2.17 (Modus ponens) The rule modus ponens is admissible in $G 3_{p f}$ and $G 3_{n f}$.

Proof From the following derivation, by using Theorem 2.10.

$$
\frac{A, A \rightarrow B \Rightarrow B, A \quad B, A, A \rightarrow B \Rightarrow B}{A, A \rightarrow B \Rightarrow B} \mathrm{~L} \rightarrow
$$


Lemma 2.18 (Replacement generalization) For any formula $A$ it is derivable that $s=t, A, \Gamma \Rightarrow \Delta, A[t / / s]$.

Proof By induction on the weight of $A$. Note that when $A$ does not contain $s$ this is an instance of Lemma 2.6, that $(C \circ D)[t / / s]$ is the same as $C[t / / s] \circ D[t / / s]$ (straightforward), and that $(\forall x A)[t / / s]$ is the same as $\forall x(A[t / / s])$ (given that by Lemma 2.4 we can keep free and bound individual variables separate).

Basic case.

$$
\frac{s=t, P, P[t / / s], \Gamma \Rightarrow \Delta, P[t / / s]}{s=t, P, \Gamma \Rightarrow \Delta, P[t / / s]}={ }_{R e p l}
$$

Inductive case. We illustrate for one case of a connective and one case of a quantified sentence, since all the cases are fairly similar.

$$
\begin{aligned}
& \begin{array}{cc}
\frac{\vdots}{s=t, C, \Gamma \Rightarrow \Delta, C[t / / s], D[t / / s]} \text { i.h. } & \frac{\vdots}{s=t, D, \Gamma \Rightarrow \Delta, C[t / / s], D[t / / s]} \\
\frac{\mathrm{R} \vee h .}{s=t, C, \Gamma \Rightarrow \Delta, C[t / / s] \vee D[t / / s]} \mathrm{R} & \frac{s=t, D, \Gamma \Rightarrow \Delta, C[t / / s] \vee D[t / / s]}{s=t, C \vee D, \Gamma \Rightarrow \Delta, C[t / / s] \vee D[t / / s]} \mathrm{L} \vee
\end{array} \\
& \frac{\vdots}{\frac{A[d / x], E ! d, s=t, \forall x A, \Gamma \Rightarrow \Delta, A[t / / s][d / x]}{E ! d, s=t, \forall x A, \Gamma \Rightarrow \Delta, A[t / / s][d / x]}} \mathrm{L} \forall \forall
\end{aligned}
$$

Note that due to the freshness condition and Lemma 2.4, $A[t / / s][d / x]$ is the same as $A[d / x][t / / s]$.

We can show that it holds for $G 3_{p f}$ that

Theorem 2.19 All of the PFL axioms Al-A6 are derivable in G3 $p$.

And then for $G 3_{n f}$ that

Theorem 2.20 All of the NFL axioms Al-A7 are derivable in $G 3_{n f}$.

We demonstrate these for each axiom. Since none of the proofs are particularly involved, their presentation is deferred to Appendix B. Instead, we proceed to prove a few interesting results specific to NFL. We start by showing that the axiom A6 of PFL does not hold in $G 3_{n f}$ :

Lemma $2.21 \Rightarrow t=t$ is not derivable in $G 3_{n f}$.

Proof By Corollary 2.11, every formula occurring in the derivation of $\Rightarrow t=t$ is either $t=t$ or atomic. But no application of the rules will remove every instance of those from the antecedent. Therefore, there is no derivation of $\Rightarrow t=t$ in $G 3_{n f}$. 
Lemma 2.22 In $G 3_{n f}$ the sentences $E ! t$ and $t=t$ are equivalent.

Proof We show this by two simple derivations:

$$
\frac{t=t, E ! t \Rightarrow t=t}{E ! t \Rightarrow t=t}=\operatorname{Ref} \quad \frac{E ! t, t=t \Rightarrow E ! t}{t=t \Rightarrow E ! t} E !
$$

Lemma 2.23 Indiscernibility of non-existents, $\neg E ! t \wedge \neg E ! s \Rightarrow A \rightarrow A[t / / s]$, is derivable in $G 3_{n f}$.

Proof By induction on the weight of $A$ (note that $A[s]$ and $A[t / / s]$ always have the same weight) we show that $\neg E ! t \wedge \neg E ! s, A \Rightarrow A[t / / s]$. If $A$ does not contain $s$, this is just an instance of Lemma 2.6.

Basic case.

$$
\begin{gathered}
\frac{E ! s, \neg E ! t, P[s] \Rightarrow P[t / / s], E ! s}{\neg E ! t, P[s] \Rightarrow P[t / / s], E ! s} E ! \\
\frac{\neg E ! t, \neg E ! s, P[s] \Rightarrow P[t / / s]}{\neg} \mathrm{L} \\
\neg E ! t \wedge \neg E ! s, P[s] \Rightarrow P[t / / s]
\end{gathered}
$$

Inductive case. Whenever some subformula $B$ does not contain $s$, an instance of the inductive hypothesis for it becomes an instance of Lemma 2.6. Note for the following proof that $C[s]$ is $C[t / / s][s / t]$, which is a special case of $C[t / / s][s / / t]$.

$$
\begin{aligned}
& \frac{\frac{\vdots}{\neg E ! t \wedge \neg E ! s, C[t / / s] \Rightarrow C[s]}}{\neg E ! t \wedge \neg E ! s, \neg C[s] \Rightarrow \neg C[t / / s]} \mathrm{L} \neg, \mathrm{R} \neg \\
& \begin{array}{cc}
\frac{\vdots}{\neg E ! t \wedge \neg E ! s, C[s], D[s] \Rightarrow C[t / / s]} i . h . & \frac{\vdots}{\neg \wedge} \\
\frac{\neg E ! t \wedge \neg E ! s, C[s] \wedge D[s] \Rightarrow C[t / / s]}{\neg} & \frac{\neg E ! t \wedge \neg E ! s, C[s], D[s] \Rightarrow D[t / / s]}{2 . h .} \mathrm{L} \wedge \\
\neg E ! t \wedge \neg E ! s, C[s] \wedge D[s] & \Rightarrow C[t / / s] \wedge D[t / / s]
\end{array}
\end{aligned}
$$

Similar for other connectives.

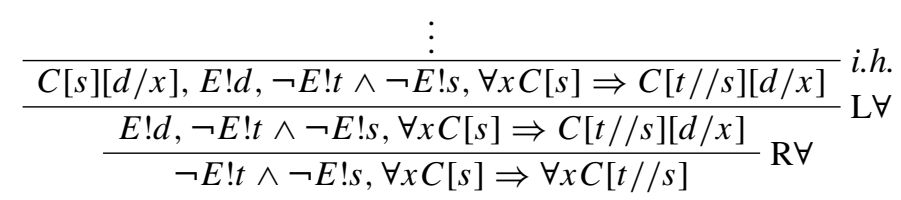

Similar for $\exists x C$.

This, and Lemmas 2.13 and 2.14, suffice to show that our formalization constitutes an adequate representation of the two systems of free logics, both in what is and what is not (in particular in the case of NFL) derivable. 


\section{Semantics}

Naturally, since we do not intend to use the axioms as semantics, we will in this section present a system of semantics. The central idea is to separate two distinct elements of semantics, namely the assignment of truth-values to atoms and to complex formulas, and abstract away from the former. In this way, we represent the semantics in purely syntactic terms, which allows us to achieve the required meta-theoretical properties in a streamlined manner.

\subsection{Generalized Semantics}

The usual exposition of semantics consists of two steps - giving a decision procedure for the assignment of truth values to atoms, and inductive rules for assignments of truth values to any other formula. However, since we ever only deal with descriptions of semantic models, i.e. their syntactic superstructure, here we will employ generalized semantics, which will suffice for the meta-theoretic proofs. We simply assume the existence of a method for assigning values to atoms, while focusing on the second step of the exposition (cf. [19, p. 81]).

Definition 3.1 (Negative structure $\mathcal{S}_{n}$ ) A negative structure $\mathcal{S}_{n}$ is a pair $\langle\mathcal{D}, \varphi\rangle$, where $\mathcal{D}=a_{1}, \ldots, b_{1}, \ldots$ countable list of free individual variables, and $\varphi$ an interpretation function on $\mathcal{L}$ :

- $\varphi(t)=t$, where $t \in \mathcal{D}$ (to emphasize its dual role we will abuse the notation slightly and write $\mathcal{D}$ as $\varphi(\mathcal{D}))$

$-\varphi(E !) \subseteq \mathcal{D}$

$-\varphi(=)=\operatorname{Ref} \cup I d$, closed under symmetry and transitivity, where

- $\operatorname{Ref}=\{\langle t, t\rangle \mid t \in \varphi(E !)\}$

- $I d \subseteq \varphi(E !) \times \varphi(E !)$

- $\varphi\left(P^{n}\right) \subseteq \varphi(E !)^{n}$ such that if $\langle s, t\rangle \in \varphi(=)$, then $\left\langle\ldots, s_{i}, \ldots\right\rangle \in \varphi\left(P^{n}\right)$ iff $\left\langle\ldots, t_{i}, \ldots\right\rangle \in \varphi\left(P^{n}\right)$, for any $n$ and any $1 \leq i \leq n$.

A positive structure is defined as

Definition 3.2 (Positive structure $\mathcal{S}_{p}$ ) The positive structure $\mathcal{S}_{p}$ differs from $\mathcal{S}_{n}$ only in that

- $\operatorname{Ref}=\{\langle t, t\rangle \mid t \in \varphi(\mathcal{D})\}$,

- $\quad I d \subseteq \varphi(\mathcal{D}) \times \varphi(\mathcal{D})$ and

$-\varphi\left(P^{n}\right) \subseteq \varphi(\mathcal{D})^{n}$.

We omit the clause $\varphi(E !) \subseteq \mathcal{D}$.

The reason we can omit $\varphi(E !) \subseteq \mathcal{D}$ is that $E$ ! is defined like any other unary predicate by the last clause of the definition. This means that $E$ ! could here be regarded 
as a non-logical predicate. As another consequence of this definition, if $s=t$ then $E ! s$ iff $E ! t$.

From these two structures we can likewise define a classical structure:

Definition 3.3 (Structure $\mathcal{S}_{c}$ ) A classical structure $\mathcal{S}_{c}$ is any structure such that it both an $\mathcal{S}_{p}$ and an $\mathcal{S}_{n}$.

Definition 3.4 (Valuation $\mathcal{V}$ ) The truth-value assignment $\mathcal{V}$ on the structure $\langle\mathcal{D}, \varphi\rangle$ is defined as

$-\mathcal{V}\left(P^{n}\left(t_{1}, \ldots, t_{n}\right)\right)=\top$ iff $\left\langle t_{1}, \ldots, t_{n}\right\rangle \in \varphi\left(P^{n}\right)$, and $\perp$ otherwise.

- Standard for connectives.

- $\mathcal{V}(\forall x A)=\top$ iff for every $t \in \varphi(E$ !) it holds that $\mathcal{V}(A[t / x])=\top$, and $\perp$ otherwise.

- $\mathcal{V}(\exists x A)=\top$ iff for some $t \in \varphi(E$ !) it holds that $\mathcal{V}(A[t / x])=\top$, and $\perp$ otherwise.

Definition 3.5 (Validity) A formula is valid on a structure iff it is true on that structure. A formula is valid iff it is valid on any structure.

\subsection{Relationship Between PFL, NFL and Classical Logic}

In any of the three definitions above we can further specify that they are non-inclusive by the addition of the condition $\varphi(E !) \neq \varnothing$. In this way, the generalized semantics systematizes the relation between classical, negative and positive structures (marked below as $\mathrm{C}, \mathrm{N}$ and $\mathrm{P}$ respectively) on both inclusive and non-inclusive versions (marked below with subscripts $i$ and $n$ respectively). For the purposes of this schematization we take it that what is usually thought of as the domain is here designated by $E$ ! (and not $\mathcal{D}$ ). This is reinforced by the axioms A3 and A4 (and Theorems 2.19 and 2.20), and some more evidence towards this claim will be provided later in the section on modalities. However, the framework here is also perfectly compatible with the single-domain semantics for positive free logics [1] (note that in Definition 3.2 we can regard $E$ ! as just an ordinary predicate).

$$
\begin{aligned}
& \mathbf{N}_{i}: \varphi\left(P^{n}\right) \subseteq \varphi(E !)^{n}, \text { Ref }=\{\langle t, t\rangle \mid t \in E !\} \\
& \mathbf{P}_{i}: \varphi\left(P^{n}\right) \subseteq \varphi(\mathcal{D})^{n}, \text { Ref }=\{\langle t, t\rangle \mid t \in \mathcal{D}\} \\
& \mathbf{C}_{i}: \varphi\left(P^{n}\right) \subseteq \varphi(\mathcal{D})^{n}, \operatorname{Ref}=\{\langle t, t\rangle \mid t \in \mathcal{D}\}, \varphi(E !)=\varphi(\mathcal{D}) \\
& \mathbf{N}_{n}: \varphi(E !) \neq \emptyset, \varphi\left(P^{n}\right) \subseteq \varphi(E !)^{n}, \operatorname{Ref}=\{\langle t, t\rangle \mid t \in E !\} \\
& \mathbf{P}_{n}: \varphi(E !) \neq \emptyset, \varphi\left(P^{n}\right) \subseteq \varphi(\mathcal{D})^{n}, \operatorname{Ref}=\{\langle t, t\rangle \mid t \in \mathcal{D}\} \\
& \mathbf{C}_{n}: \varphi(E !) \neq \emptyset, \varphi\left(P^{n}\right) \subseteq \varphi(\mathcal{D})^{n}, \operatorname{Ref}=\{\langle t, t\rangle \mid t \in \mathcal{D}\}, \varphi(E !)=\varphi(\mathcal{D})
\end{aligned}
$$

Each inclusive structure is obtained from its non-inclusive counterpart, by extending the interpretation of $E$ ! to empty cases.

A positive structure is obtained from a negative structure when $\operatorname{Ref}$ and $\varphi\left(P^{n}\right)$ are extended from members of $E$ ! to members of $\mathcal{D}$. Finally, a classical structure is obtained from the positive one by extending the interpretation of $E$ ! to all members of $\mathcal{D}$ (Fig. 4). 
Fig. 4 Relationship between PFL, NFL and CL

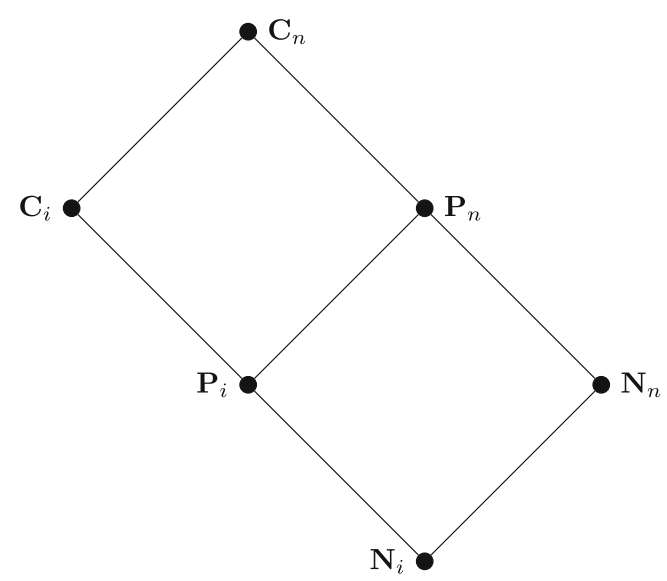

Of these, the structure $C_{n}$ marks the classical logic. Here, the domain is not empty, and all the free individual variables refer (since in a negative structure $t=t$ holds only if $E ! t$, and in a positive one $t=t$ holds for every $t$ ).

While it is a classical structure, $C_{i}$ is not the classical logic. Even though it is here mostly a theoretical construct, it does correspond to an empty logic (more on that in Section 5.2).

\section{Meta-theoretical Properties}

We now proceed to show that the systems $G 3_{p f}$ and $G 3_{n f}$ are sound and complete with respect to their appropriate generalized semantics.

\subsection{Soundness}

Definition 4.1 A sequent $\Gamma \Rightarrow \Delta$ is valid on a structure $\mathcal{S}$ iff whenever all formulas in $\Gamma$ are valid on $\mathcal{S}$, some formula in $\Delta$ also is.

We prove that

Theorem 4.2 If a sequent $\Gamma \Rightarrow \Delta$ is derivable in $G 3_{p f}\left(G 3_{n f}\right)$, it is valid on any structure $\mathcal{S}_{p}\left(\mathcal{S}_{n}\right)$.

Proof By induction on the height of the derivation of $\Gamma \Rightarrow \Delta$. Straightforward for the basic case, and simple for identity, $E$ !, and connectives. We illustrate on an example.

If the last step of the derivation is obtained by $L \wedge$, then it has the form

$$
\frac{C, D, \Gamma \Rightarrow \Delta}{C \wedge D, \Gamma \Rightarrow \Delta} \mathrm{L} \wedge
$$

Assume $C \wedge D$ and all the formulas in $\Gamma$ are valid. Then by Definition 3.4, $C, D, \Gamma$ are all valid. Then by inductive hypothesis, some formula in $\Delta$ is valid. 
If the last step of the derivation is obtained by $\mathrm{R} \wedge$, then it has the form

$$
\frac{\Gamma \Rightarrow \Delta, C \quad \Gamma \Rightarrow \Delta, D}{\Gamma \Rightarrow \Delta, C \wedge D} \mathrm{R} \wedge
$$

Assume all the formulas in $\Gamma$ are valid. Then by inductive hypothesis, either some formula in $\Delta$ is valid, in which case so is some formula in $\Delta, C \wedge D$, or both $C$ and $D$ are, in which case by Definition 3.4 so is $C \wedge D$.

If the last step of the derivation is obtained by $L \forall$, then it has the form

$$
\frac{C[t / x], E ! t, \forall x C, \Gamma \Rightarrow \Delta}{E ! t, \forall x C, \Gamma \Rightarrow \Delta} \mathrm{L} \forall
$$

Assume $E ! t, \forall x C$ and all the formulas in $\Gamma$ are valid. Then, since $\forall x C$ and $E ! t$ are valid, by Definition 3.4 so is $C[t / x]$, therefore all of $C[t / x], E ! t, \forall x C, \Gamma$ are valid, and by the inductive hypothesis so is some formula in $\Delta$.

If the last step of the derivation is obtained by $\mathrm{R} \forall$, then it has the form

$$
\frac{E ! t, \Gamma \Rightarrow \Delta, C[t / x]}{\Gamma \Rightarrow \Delta, \forall x C} \mathrm{R} \forall
$$

Assume all the formulas in $\Gamma$ are valid and neither any formula in $\Delta$ nor $\forall x C$ are. So by Definition 3.4 there is a free individual variable $t$ such that $E$ ! $t$ is valid, but $C[t / x]$ isn't. Let that variable be $t$. So, none of the formulas in $\Delta, C[t / x]$ are valid, and therefore by contraposition of the inductive hypothesis, either some formula in $\Gamma$ or $E ! t$ isn't. Contradiction either way, and so either some formula in $\Delta$ or $\forall x C$ is valid.

Parallel for $\mathrm{R} \exists$ and $\mathrm{L} \exists$ (for the latter the proof is direct).

\subsection{Completeness}

We start by the definition of a reduction tree. Intuitively, this represents a bottom-up proof search.

Definition 4.3 (Reduction tree) A reduction tree for a sequent $\Gamma \Rightarrow \Delta$ is built in steps. At step 0 , the tree is just $\Gamma \Rightarrow \Delta$. Any sequent that does not contain the same atomic formula in both the antecedent and the consequent is called active.

Each subsequent step consists of stages. At each stage and for each sequent $\Gamma_{i} \Rightarrow \Delta_{i}$ active at the beginning of it, we apply to any eligible (pair of) formulas in the sequent the rule of the stage once (thereby extending the height of the tree by $n$, for $n$ such formulas in $\Gamma_{i} \Rightarrow \Delta_{i}$, and creating at most $2^{n}$ branches, before proceeding to the next stage). We call an application of a rule to the formula(s) their reduction.

The order of stages is:

(1) $\mathrm{L} \wedge$ (2) $\mathrm{R} \wedge$ (3) $\mathrm{L} \vee(4) \mathrm{R} \vee(5) \mathrm{L} \neg$ (6) $\mathrm{R} \neg$ (7) $\mathrm{L} \rightarrow$ (8) $\mathrm{R} \rightarrow$

(9) $\mathrm{L} \forall$, for every pair of formulas $\forall x A$ and $E ! t$ in $\Gamma_{i}$.

(10) $\mathrm{R} \forall$, taking for the reduction of each formula $\forall x A$ in $\Delta_{i}$ from the denumerable list of free individual variables the first such variable $t$ not yet used in the reduction tree.

(11) $\mathrm{L} \exists$, treated symmetrically to $\mathrm{R} \forall$ (12) $\mathrm{R} \exists$, treated symmetrically to $\mathrm{L} \forall$ 
$(13)={ }_{R e p l}(14)=_{R e f}$, in the case of $G 3_{p f}$ for any $t$ such that it occurs in $\Gamma_{i} \Rightarrow \Delta_{i}$ but $t=t$ does not occur in $\Gamma_{i}$,

(15) $E$ !, for $G 3_{n f}$ only For each active sequent to which no rule can be applied, we just copy it.

We now show that

Lemma 4.4 For any sequent $\Gamma \Rightarrow \Delta$ its reduction tree either produces a proof or it produces a structure and a valuation that validates all the formulas in $\Gamma$ and none of the formulas in $\Delta$.

Proof It is clear that a reduction tree with no active sequents will produce, read top down (and thus beginning with initial sequents and ending with $\Gamma \Rightarrow \Delta$ ), a finite derivation of that sequent. The second part is more involved and goes through several lemmas below.

We now build an invalidating structure and a valuation from a reduction tree to prove the second part. The existence of an infinite branch is guaranteed by the König's lemma in the usual way [19, p. 82].

Definition 4.5 (Refutation structure $\mathcal{C}$ ) Take an infinite branch

$$
\Pi \equiv \Gamma_{0} \Rightarrow \Delta_{0}, \ldots, \Gamma_{i} \Rightarrow \Delta_{i}, \ldots
$$

of a reduction tree for a sequent $\Gamma \Rightarrow \Delta$ (where $\Gamma_{0} \Rightarrow \Delta_{0}$ is $\Gamma \Rightarrow \Delta$ ) and consider sets $\Gamma^{*} \equiv \bigcup \Gamma_{i}$ and $\Delta^{*} \equiv \bigcup \Delta_{i}$ for $0 \leq i$. A refutation structure $\mathcal{C}$ for a sequent $\Gamma \Rightarrow \Delta$ is built by assigning $\top$ to all atomic formulas in $\Gamma^{*}$ and $\perp$ to all other atomic formulas (therefore including all atomic formulas in $\Delta^{*}$ ), and otherwise the same as $\mathcal{V}$.

We first show that this produces the correct type of structure. Since the proof for PFL is very similar, we will here focus on NFL.

Lemma 4.6 The refutation structure $\mathcal{C}$ for a sequent $\Gamma \Rightarrow \Delta$ in $G 3_{n f}$ is a structure $\mathcal{S}_{n}$. Specifically, (i) E! $t$ implies $t \in \mathcal{D}$ (ii) $E ! t \rightarrow t=t$ (iii) $s=t \rightarrow(E ! s \wedge E ! t)$, (iv) $P_{n}\left(\ldots, t_{i}, \ldots\right) \rightarrow E ! t_{i}$ and $(v)$ if $s=t$ then $P_{n}\left(\ldots, s_{i}, \ldots\right)$ iff $P_{n}\left(\ldots, t_{i}, \ldots\right)$.

Proof By examining Definition 4.3.

(i) Immediate from the fact all formulas in $\Gamma \Rightarrow \Delta$ are formulas of $\mathcal{L}$ and the procedure for selecting free individual variables in Definition 4.3.

(ii) By stage (14), for every atomic formula $E ! t$ in $\Gamma^{*}, t=t$ is in $\Gamma^{*}$. Therefore by Definition 4.5, $t=t$ is likewise true.

(iii) By stage (15), for any atomic formula in $\Gamma^{*}$, including $s=t$, the formulas $E$ ! $s$ and $E ! t$ are likewise in $\Gamma^{*}$ and therefore true by Definition 4.5.

(iv) Similar to (iii).

(v) Assume that $s=t$ and $P_{n}\left(\ldots, s_{i}, \ldots\right)$ are in $\Gamma^{*}$. By stage (13), so is $P_{n}\left(\ldots, t_{i}, \ldots\right)$, and therefore true by Definition 4.5 . Same for the other direction. 
Now we show that

Lemma 4.7 Any formula A occurring in $\Gamma^{*}$ is assigned $\top$ by the refutation structure $\mathcal{C}$ and any formula $B$ occurring in $\Delta^{*}$ is assigned $\perp$ by the refutation structure $\mathcal{C}$.

Proof By simultaneous induction on the weight of $A$ and $B$.

Basic step. Immediate from Definition 4.5 and noting that $\Gamma^{*}$ and $\Delta^{*}$ share no atoms.

Inductive step. We illustrate for one example of a connective, as all are straightforward.

$(\wedge)$ If $A$ is a formula $C \wedge D$, then by stage (1) of Definition 4.3, $C$ and $D$ are also in $\Gamma^{*}$, and by inductive hypothesis assigned $\top$, and so $C \wedge D$ is $\top$.

If $B$ is a formula $C \wedge D$, then by stage (2) of Definition 4.3, either $C$ or $D$ are also in $\Delta^{*}$, and by inductive hypothesis assigned $\perp$, and so $C \wedge D$ is $\perp$.

Similar for other connectives.

( $\forall$ ) If $A$ is a formula $\forall x C$, then by stage (9) of Definition 4.3, for every $t \in D$, if $E ! t$ is in $\Gamma^{*}$ (and by inductive hypothesis $\top$ ) then $C[t / x]$ is in $\Gamma^{*}$ (and by inductive hypothesis $\top$ ), and so $\forall x C$ is $\top$.

If $B$ is a formula $\forall x D$, then by stage (10) of Definition 4.3, for some $t \in D$, $E ! t$ is in $\Gamma^{*}$ (and by inductive hypothesis $T$ ) and $D[t / x]$ is in $\Delta^{*}$ (and by inductive hypothesis $\perp$ ), and so $\forall x D$ is $\perp$.

Similar for $\exists$.

Finally, we have that

Theorem 4.8 If $\Gamma \Rightarrow \Delta$ is valid in NFL or PFL, then $\Gamma \Rightarrow \Delta$ is derivable in $G 3_{n f}$ or $G 3_{p f}$, respectively.

Proof By contraposition we prove that if a sequent is not derivable, the entailment doesn't hold. Immediate from Lemmas 4.4 and 4.7. Specifically, by the latter we know that all the formulas of $\Gamma$ are true, but none of $\Delta$ are.

The proof for $G 3_{p f}$ is nearly identical.

Observation 4.9 Note that the result in the Theorems 4.2 and 4.8, combined with the results in Theorems 2.19 and 2.20, demonstrate the adequacy of the generalized semantics.

\section{Further Varieties}

In this section we observe some further varieties of $G 3_{p f}$ and $G 3_{n f}$ mentioned in the Section 3.2. We first note that the two systems described so far are compatible with an empty domain. We then add a rule preventing that, and briefly discuss the resulting (non-inclusive) logics. Furthermore, we also take a look at the logic corresponding to the structure $C_{i}$. 


\subsection{Non-inclusive Logics}

Note that for the sequent $\forall x A \Rightarrow \exists x A$ the refutation structure $\mathcal{C}$ will be generated by an infinite branch containing only that sequent, and the truth condition for $\forall$ will be vacuously fulfilled as no $E ! t$ appears as true in $\Gamma^{*}$. So nothing we have said so far actually excludes the possibility of $\varphi\left(E\right.$ !) being empty in $G 3_{p f}$ and $G 3_{n f}$, i.e. of them being inclusive logics. We can make them explicitly non-inclusive by the addition of the rule:

$$
\frac{E ! t, \Gamma \Rightarrow \Delta}{\Gamma \Rightarrow \Delta} \mathrm{NI}, t \text { fresh }
$$

This rule makes "something exists" a theorem:

Lemma $5.1 \Rightarrow \exists x E ! x$

Proof

$$
\frac{E ! t \Rightarrow \exists x E ! x, E ! t}{E ! t \Rightarrow \exists x E ! x} \mathrm{R} \exists
$$

This rule is not necessary in the classical system $G 3_{p f+n f}$, which contains all the rules of both G3 $3_{p f}$ and $\mathrm{G} 3_{n f}$ (and by inspection of their proofs also all the structural properties), since it can be easily shown that

Theorem 5.2 In a system $G 3_{p f+n f}$, the rule NI is admissible.

Proof

$$
\begin{array}{cl}
\frac{E ! t, \Gamma \Rightarrow \Delta}{t=t, E ! t, \Gamma \Rightarrow \Delta} & \text { Lemma } 2.7 \\
\frac{t=t, \Gamma \Rightarrow \Delta}{\Gamma \Rightarrow \Delta} & E ! \\
\text { Ref }
\end{array}
$$

This derivation mirrors the semantical explanation of the interaction of the two systems from the Section 3.2. Of course, nothing we have said so far excludes the list of free individual variables $\mathcal{D}$ being empty either. Without that assumption, we obtain the logic $G 3 C_{i}$ which corresponds to an empty logic.

\subsection{Empty Logic $G 3 C_{i}$}

If we assume $\mathcal{D}$ is empty, $\forall x A$ becomes an abbreviation for $T$ (see [23]). To represent this semantics in terms of a sequent calculus any formula $P^{n}\left(t_{1}, \ldots, t_{n}\right)$ can be seen simply as an abbreviation for $\perp$ (for the sake of simplicity we exclude 0 ary predicates from the language). Therefore, the rules $E !,=_{\text {Ref }}$ and $=_{R e p l}$ reduce to contraction, which can be shown admissible. Furthermore, every initial sequent is simply an instance of the rule $\mathrm{L} \perp$. The system then has the following form: 
Definition 5.3 (Empty logic $G 3 C_{i}$ ) The empty logic $G 3 C_{i}$ is obtained from $G 3_{n f}$ by removing the rules for initial sequents, $E !,={ }_{\text {Ref }}$ and $=_{R e p l}$ and replacing the quantifier rules by all the rules in Fig. 5 .

Equivalently, this logic can be obtained by the same procedure from $G 3_{p f}$, (with the rule $E$ ! vacuously removed).

Structural rules for this logic are demonstrated in a routine manner, so we will limit ourselves here to just showing the admissibility of contraction, as it was used in the preceding paragraph.

Lemma 5.4 (Contraction in $G 3 C_{i}$ ) Contraction is height-preserving admissible in $G 3 C_{i}$. Namely,

i) If $\vdash_{n} C, C, \Gamma \Rightarrow \Delta$ then $\vdash_{n} C, \Gamma \Rightarrow \Delta$.

ii) If $\vdash_{n} \Gamma \Rightarrow \Delta, C, C$ then $\vdash_{n} \Gamma \Rightarrow \Delta, C$.

Proof Simultaneous for (i) and (ii) by induction on the height of the derivation.

In the initial case (for zero-premise rules since there are no initial sequents), if $C, C, \Gamma \Rightarrow \Delta\left(\vdash_{n} \Gamma \Rightarrow \Delta, C, C\right)$ is a conclusion of a zero-premise rule, then so is $C, \Gamma \Rightarrow \Delta(\Gamma \Rightarrow \Delta, C)$.

Standard for propositional rules.

For $\mathrm{L} \forall$ and $\mathrm{R} \exists$ we apply the inductive hypothesis to the upper sequent of the rule and then the rule in a routine way.

Like this case, all other properties of the system are straightforward and routine, and will be skipped.

\subsubsection{Adequacy of $\mathrm{G}^{3} \mathrm{C}_{i}$}

One axiomatization by which we can measure the adequacy of our formalization is that in [7]. The notation is adjusted to be more in line with the current standards and the present paper.

Bottom rule:

$$
\overline{\perp, \Gamma \Rightarrow \Delta} \mathrm{L} \perp
$$

Quantifier rules:

$$
\begin{aligned}
& \frac{\forall x A, \Gamma \Rightarrow \Delta}{\Gamma \Rightarrow \Delta} \mathrm{L} \forall \quad \overline{\Gamma \Rightarrow \Delta, \forall x A} \mathrm{R} \forall \\
& \overline{\exists x A, \Gamma \Rightarrow \Delta} \mathrm{L} \exists \quad \frac{\Gamma \Rightarrow \Delta, \exists x A}{\Gamma \Rightarrow \Delta} \mathrm{R} \exists
\end{aligned}
$$

Fig. 5 G3C ${ }_{i}$ 
Definition 5.5 (Empty logic QE)

1. If $A$ is tautologous, then $\vdash A$

2. $\forall x \forall y A \rightarrow \forall y \forall x A^{1}$

3. $\forall x(A \rightarrow B) \rightarrow(\forall x A \rightarrow \forall x B)$

4. $A \rightarrow \forall x A$, if $x$ is not free in $A$

5. $\forall x A \rightarrow \forall y A[y / x]$

6. $(A \rightarrow \forall x B) \rightarrow \forall x(A \rightarrow B)$, if $x$ is not free in $A$

7. If $\vdash A$ and $\vdash A \rightarrow B$ then $\vdash B$.

Theorem 5.6 All of the Axioms 1-7 of $Q E$ are derivable in $G 3 C_{i}$.

Proof Note that the propositional part of $G 3 C_{i}$ is simply standard G3 classical propositional logic, so (1) follows from completeness of it. (2)-(6) are easily derivable from $\mathrm{R} \forall$ and $\mathrm{R} \rightarrow$. Finally, (7) follows from Lemma 2.17 (again noting that the propositional part of $G 3 C_{i}$ is standard).

It would be interesting to check whether this axiomatization is complete with respect to the intended semantics, as it is not obvious to us the axiomatization can be used to prove anything other than universal closures of tautologies (as opposed to any universally quantified formula). However, such a discussion would take us too far off topic since, as stated previously, we are here to a large extent dealing with an artifact of the systematization.

\section{Modal Logic}

When we move to the modal context, the principle of unrestricted specification, $\forall x A \rightarrow A[t / x]$, fails to hold in general [20, pp. 223-224]. Therefore, it seems prima facie that free and modal logics might be a good fit (cf. [5, 12]). In fact, as we will see in this section, not only is there structural correspondence between modal and quantifier rules (this is by design), but the extension of one into the other is exceedingly straightforward.

The modal logic system presented here will be an extension of the base systems $G 3_{p f}$ and $G 3_{n f}$ into labeled sequent calculi, following $[16,20]$, with the addition of the modal operator rules.

Definition 6.1 $\left(G 3 K_{p f}, G 3 K_{n f}\right)$ We add a countable set of labels $w_{1}, \ldots$,

$w_{n}, \ldots$ to our language, extend it with relational formulas of the form $w R o$ and replace every formula of the form $A$ with a labeled formula of the form $w: A$. Moreover, we replace every side and principal formula $A$ in the rules of $G 3_{p f}$ and $G 3_{n f}$ by a labeled formula $w: A$ (Fig. 6). Finally, we add the modal operator rules (Fig. 7).

${ }^{1}[23]$ points out, citing [3], that this axiom is easily omitted. 
Quantifier rules:

$$
\begin{aligned}
& \frac{w: A[t / x], w: E ! t, w: \forall x A, \Gamma \Rightarrow \Delta}{w: E ! t, w: \forall x A, \Gamma \Rightarrow \Delta} \mathrm{L} \forall \quad \frac{w: E ! t, \Gamma \Rightarrow \Delta, w: A[t / x]}{\Gamma \Rightarrow \Delta, w: \forall x A} \mathrm{R} \forall^{*} \\
& \frac{w: E ! t, w: A[t / x], \Gamma \Rightarrow \Delta}{w: \exists x A, \Gamma \Rightarrow \Delta} \mathrm{L} \exists * \quad \frac{w: E ! t, \Gamma \Rightarrow \Delta, w: \exists x A, w: A[t / x]}{w: E ! t, \Gamma \Rightarrow \Delta, w: \exists x A} \mathrm{R} \exists \\
& * t \text { fresh. }
\end{aligned}
$$

Fig.6 Quantifier rules for $\mathrm{G}_{3} \mathrm{~K}_{p f}, \mathrm{G} \mathrm{K}_{n f}$

Note that the labeled formula $w: A$ is a formula of the new language iff $A$ was a formula of the old one. Therefore, sentence formation rules do not apply to relational formulas (i.e. they are always atomic, and never active in logical rules).

Should we wish to also extend the systems with the explicit rule for noninclusiveness NI, the resulting rule would correspond to the non-emptiness rule from [20].

The interesting case here are the new quantifier rules, which will now have the following form:

Note that the usual extension of the quantifier rules into a modal context requires us to specify that $t$ is an element of the domain of $w$ [20]. No such provision is required here due to the presence of the formula $E ! t$. Therefore, all that is required to transform the rules is adding the same label to all the active and principal formulas of the rules. The modal operator rules are:

\subsection{Structural Properties}

Given that the non-modal rules have been minimally modified, it is easy to see that Lemmas 2.6 - 2.9, as well as Theorem 2.10 will still hold, so we just need to extend those with the case of modal rules. Moreover, since the modal rules closely resemble

Modal rules:

$$
\begin{aligned}
& \frac{o: A, w: \square A, w R o, \Gamma \Rightarrow \Delta}{w: \square A, w R o, \Gamma \Rightarrow \Delta} \mathrm{L} \square \quad \frac{w R o, \Gamma \Rightarrow \Delta, o: A}{\Gamma \Rightarrow \Delta, w: \square A} \mathrm{R}^{*} \\
& \frac{w R o, o: A, \Gamma \Rightarrow \Delta}{w: \diamond A, \Gamma \Rightarrow \Delta} \mathrm{L} \diamond * \quad \frac{w R o, \Gamma \Rightarrow \Delta, w: \diamond A, o: A}{w R o, \Gamma \Rightarrow \Delta, w: \diamond A} \mathrm{R} \diamond \\
& \text { * } o \text { fresh }
\end{aligned}
$$

Fig. 7 Modal rules for $\mathrm{G}_{3} \mathrm{~K}_{p f}, \mathrm{G}_{3} \mathrm{~K}_{n f}$ 
the quantifier ones, the proofs there will be quite similar. Therefore, we simply note that

Theorem 6.2 Axiom generalization, height-preserving weakening and contraction, as well as cut are admissible in $G 3 K_{p f}$ and $G 3 K_{n f}$. Moreover, the rules of the systems are (height-preserving) invertible.

\subsection{Generalized Semantics}

In developing the generalized semantics for $G 3 K_{p f}$ and $G 3 K_{n f}$ we adopt standard Kripke semantics, and define two different types of frames, depending on whether identity is taken as invariant (marked with a superscript $I$ ) or varying (marked with $V)$. We start off with a more general type:

Definition 6.3 A negative (positive) Kripke frame $\mathcal{F}_{n}\left(\mathcal{F}_{p}\right)$ is a pair $\langle W, R\rangle$, where $W$ is a countable multiset (we note the $i$-th member of $W$ as $w_{i}$ ) of negative structures $\mathcal{S}_{n}=\langle\mathcal{D}, \varphi\rangle$ (positive structures $\mathcal{S}_{p}$ ), and $R \subseteq W \times W$.

This frame allows for lists of names that vary between worlds. If we want to keep them fixed between worlds, we define the frame

Definition 6.4 A negative (positive) Kripke frame $\mathcal{F}_{n}^{V}\left(\mathcal{F}_{p}^{V}\right)$ is a tuple $\langle\mathcal{D}, W, R\rangle$, where $\mathcal{D}=a_{1}, \ldots, b_{1}, \ldots$ as before, $W$ is a countable multiset of negative (positive) interpretation functions $\varphi$, and $R \subseteq W \times W$.

Now the list of names is fixed between worlds, but all predicates, including identity, are interpreted separately in each. Since identity can vary, we designate the frames with $V$. To get an identity predicate that is invariant, we deflate $W$ further and obtain a frame

Definition 6.5 A negative (positive) Kripke frame $\mathcal{F}_{n}^{I}\left(\mathcal{F}_{p}^{I}\right)$ is a tuple $\left\langle\mathcal{F}_{n}^{*}, W, R\right\rangle$, $\left(\left\langle\mathcal{F}_{p}^{*}, W, R\right\rangle\right)$ where $\mathcal{F}_{n}^{*}$ is some negative structure without $\varphi\left(P^{n}\right)\left(\mathcal{F}_{p}^{*}\right.$ a positive structure without $\left.\varphi\left(P^{n}\right)\right), W$ is a countable multiset of negative interpretation functions over $n$-ary predicates $\varphi\left(P^{n}\right)$ (positive functions $\varphi\left(P^{n}\right)$ ), and $R \subseteq W \times W$. For each $\varphi_{i} \in W, \varphi_{i}(=)$ and $\varphi_{i}(E !)\left(\varphi_{i}(=)\right)$ are identical to $\varphi$ of $\mathcal{F}_{n}^{*}\left(\mathcal{F}_{p}^{*}\right)$.

Given that the unrestricted instantiation fails in models with varying domains regardless of the limitation to $E$ !, the first type of frames will not be of particular interest to us, so we focus on the other two. We call the logics defined by these successive frames $K_{\mathbf{n f}}, K_{\mathbf{p f}}, K_{\mathbf{i n f}}$ and $K_{\mathbf{i p f}}$.

In each of these cases, a model is

Definition 6.6 (Model $\mathcal{M}$ ) A model $\mathcal{M}$ is a pair $\langle\mathcal{F}, \mathcal{V}\rangle$, where $\mathcal{F}$ is some frame from Definitions $6.4-6.5$, and $\mathcal{V}$ is valuation such that

$-\mathcal{M} \Vdash_{\varphi_{i}} P^{n}\left(t_{1}, \ldots, t_{n}\right)$ iff $\left\langle t_{1}, \ldots, t_{n}\right\rangle \in \varphi_{i}\left(P^{n}\right)$, 
- Standard for connectives,

- $\mathcal{M} \Vdash_{\varphi_{i}} \forall x A$ iff for every $t$ s.t. $\mathcal{M} \Vdash_{\varphi_{i}} E ! t$ it holds that $\mathcal{M} \Vdash_{\varphi_{i}} A[t / x]$,

- $\mathcal{M} \Vdash_{\varphi_{i}} \exists x A$ iff for some $t$ s.t. $\mathcal{M} \Vdash_{\varphi_{i}} E$ ! $t$ it holds that $\mathcal{M} \Vdash_{\varphi_{i}} A[t / x]$,

- $\mathcal{M} \Vdash_{\varphi_{i}} \square A$ iff for every $\varphi_{n}$ s.t. $\left\langle\varphi_{i}, \varphi_{n}\right\rangle \in R$ it holds that $\mathcal{M} \Vdash_{\varphi_{n}} A$

- $\mathcal{M} \Vdash_{\varphi_{i}} \diamond A$ iff for some $\varphi_{n}$ s.t. $\left\langle\varphi_{i}, \varphi_{n}\right\rangle \in R$ it holds that $\mathcal{M} \Vdash_{\varphi_{n}} A$

A formula $w: A$ is valid in $\mathcal{M}=\langle\mathcal{F}, \mathcal{V}\rangle$ if $w \in \mathcal{F}$ and $\mathcal{M} \Vdash_{w} A$. A sequent $\Gamma \Rightarrow \Delta$ is valid in $\mathcal{M}$ if whenever every $w: A$ in $\Gamma$ is valid, then some $w: A$ in $\Delta$ is valid.

\subsection{Invariance of Identity}

In the sequent calculus case, to make identity invariant across worlds/ labels (whence it follows it is necessary), we add the rule of invariance of identity to $G 3 K_{p f}$ and $G 3 K_{n f}$ thereby obtaining, respectively, $G 3 K_{i p f}$ and $G 3 K_{i n f}$. The rule is adopted from [17], and we follow the authors' naming of it as the rule for rigidity.

From this rule the necessity of identity easily follows:

Lemma 6.7 Necessity of identity: $w: s=t \Rightarrow w: \square s=t$

$$
\begin{aligned}
& \text { Proof } \\
& \qquad \frac{o: s=t, w R o, w: s=t \Rightarrow o: s=t}{\frac{w R o, w: s=t \Rightarrow o: s=t}{w: s=t \Rightarrow w: \square s=t} R \square}=R i g
\end{aligned}
$$

\subsection{Meta-theoretical Properties}

In this section each of the systems $G 3 K_{p f}$, $G 3 K_{n f}$, $G 3 K_{i p f}$ and $G 3 K_{i n f}$ will be shown sound and complete with respect to the appropriate semantics.

Theorem 6.8 If a sequent $\Gamma \Rightarrow \Delta$ is derivable in $G 3 K_{p f}\left(G 3 K_{n f}\right)$, it is valid on any Kripke frame $\mathcal{F}_{p}^{V}\left(\mathcal{F}_{n}^{V}\right)$.

Proof Routine by induction on the height of the derivation with relational atoms $w R o$ valid iff $\langle w, o\rangle \in R$ and validity of a sequent mutatis mutandis as in Definition 3.5 .

$$
\frac{w: s=t, o: s=t, \Gamma \Rightarrow \Delta}{w: s=t, \Gamma \Rightarrow \Delta}=R i g
$$

Fig. 8 Invariance of identity rule 
Theorem 6.9 If a sequent $\Gamma \Rightarrow \Delta$ is derivable in $G 3 K_{\text {ipf }}\left(G 3 K_{\text {inf }}\right)$, it is valid on any Kripke frame $\mathcal{F}_{p}^{I}\left(\mathcal{F}_{n}^{I}\right)$.

Proof By induction on the height of the derivation. A new case to check is the rule $={ }_{\text {Rig }}$. If the last step of the derivation was obtained by it then it has the form:

$$
\frac{w: s=t, o: s=t, \Gamma \Rightarrow \Delta}{w: s=t, \Gamma \Rightarrow \Delta}=R i g
$$

Assume $w: s=t, o: s=t, \Gamma \Rightarrow \Delta$ is valid. Then for any model such that whenever for any $r: A$ in $\Gamma$ it holds that $\mathcal{M} \Vdash_{r} A$ and moreover that $\mathcal{M} \Vdash_{w} s=$ $t$ and $\mathcal{M} \vdash_{o} s=t$, it holds for some $r: B$ in $\Delta$ that $\mathcal{M} \vdash_{r} B$. Now assume $w: s=t, \Gamma$ is valid. But $\mathcal{M} \Vdash_{w} s=t$ iff $\mathcal{M} \Vdash_{o} s=t$ by Definition 6.5, so $w: s=t, o: s=t, \Gamma$ is likewise valid. Therefore, so is some $r: B$ in $\Delta$.

We begin the proof of completeness by extending the definition of the reduction tree:

Definition 6.10 We extend Definition 4.3 by first noting that the appropriate applications of the rules now include labeled formulas (this is straightforward) and then adding the following steps:

(16) $\mathrm{L} \square$, for every formula $w: \square A$ and every relational formula $w R o$ in $\Gamma_{i}$.

(17) $\mathrm{R} \square$, taking for the reduction of each formula $w: \square A$ in $\Delta_{i}$ from the countable list of appropriate interpretation functions the first one not yet used in the reduction tree.

(18) $\mathrm{L} \diamond$, treated symmetrically to $\mathrm{R} \square$ (19) $\mathrm{R} \diamond$, treated symmetrically to $\mathrm{L} \square$

(20) $={ }_{R i g}$, for $G 3 K_{l p f}$ and $G 3 K_{l n f}$ only, taking for the reduction of each formula $w: s=t$ every interpretation function $o$ appearing in $\Gamma_{i}$ and $\Delta_{i}$.

We build a refutation structure same as in Definition 4.5. Note that the results of the Lemma 4.6 are extended. Specifically, since relational formulas occur only in the antecedent, for each $w R o$ we add $\langle w, o\rangle \in R$ to generate an appropriate Kripke frame. Moreover, in the case of $G 3 K_{i p f}$ and $G 3 K_{\text {inf }}$, for any $w$ and $o, \mathcal{M} \Vdash_{w} s=t$ iff $\mathcal{M} \Vdash_{o} s=t$.

It is easy to see that

Lemma 6.11 The results of the Lemma 4.7 hold mutatis mutandis, and are extended for the cases of modal formulas.

Proof The first clause is straightforward (replacing $A$ with $w: A$ ). For modal formulas:

( $\square$ ) If $A$ is a formula $w: \square C$ then by stage (16) for every $o$ such that $w R o$ is in $\Gamma^{*}$ (and therefore $\langle w, o\rangle \in R$ ), $o: C$ is in $\Gamma^{*}$, and therefore by inductive hypothesis $\mathcal{M} \Vdash_{o} C$. Therefore, $\mathcal{M} \Vdash_{w} \square C$. 
If $B$ is a formula $w: \square D$ then by stage (17) for some $o$ such that $w R o$ is in $\Gamma^{*}$ (and therefore $\langle w, o\rangle \in R$ ), $o: C$ is in $\Delta^{*}$, and therefore by inductive hypothesis $\mathcal{M} \nVdash_{o} C$. Therefore, $\mathcal{M} \nVdash_{w} \square D$.

Similar for $\diamond$.

Finally, we have that

Theorem 6.12 If $\Gamma \Rightarrow \Delta$ is valid in the $K_{p f}, K_{n f}$, $K_{i p f}$ and $K_{\text {inf }}$, then it is derivable in $G 3 K_{p f}$, G3K $K_{n f}, G 3 K_{i p f}$ and $G 3 K_{i n f}$, respectively.

\subsection{Applications}

We make use of this framework to illustrate the point made earlier about the role the predicate $E$ ! plays in free logics. We first note that in frames $\mathcal{F}_{n}^{V}$ the Barcan formulas fail. As an illustration, consider the converse Barcan formula, $\square \forall x A x \rightarrow \forall x \square A x$, and a model where $\mathcal{D}=\left\{t_{1}, t_{2}\right\}, W=\left\{\varphi_{1}, \varphi_{2}\right\}, \varphi_{1}(E !)=\left\{t_{1}, t_{2}\right\}, \varphi_{2}(E !)=\left\{t_{2}\right\}$, $\varphi_{1}(A)=\left\{t_{1}, t_{2}\right\}, \varphi_{2}(A)=\left\{t_{2}\right\}$ and $R=\left\{\left\langle\varphi_{1}, \varphi_{2}\right\rangle\right\}$. Here $\mathcal{M} \Vdash_{\varphi_{2}} \forall x A x$ and therefore $\mathcal{M} \Vdash_{\varphi_{1}} \square \forall x A x$, but $\mathcal{M} \nVdash_{\varphi_{2}} A t_{1}$, so $\mathcal{M} \nVdash_{\varphi_{1}} \square A t_{1}$ and therefore $\mathcal{M} \nVdash_{\varphi_{1}} \forall x \square A x$. Note that this counterexample works for both inclusive and non-inclusive versions.

To obtain Barcan formulas we would need to add standard restrictions, applied to the predicate $E$ ! instead of the domain. Interestingly, that is not the case in frames $\mathcal{F}_{n}^{I}$. In the modal negative free logic with the invariance of identity rule added, $G 3 K_{\text {inf }}$, and corresponding to frames $\mathcal{F}_{n}^{I}$, the predicate $E$ ! behaves in the same way as identity (as shown in the Lemma that immediately follows), and therefore Barcan formulas hold:

Lemma 6.13 $w: E ! t \Rightarrow w: \square E ! t$

$$
\begin{aligned}
& \text { Proof } \\
& \qquad \frac{o: E ! t, o: t=t, w: t=t, w R o, w: E ! t \Rightarrow o: E ! t}{o: t=t, w: t=t, w R o, w: E ! t \Rightarrow o: E ! t}=R ! \\
& \frac{w: t=t, w R o, w: E ! t \Rightarrow o: E ! t}{\frac{w R o, w: E ! t \Rightarrow o: E ! t}{w: E ! t \Rightarrow w: \square E ! t} \mathrm{R} \square}
\end{aligned}
$$

Lemma 6.14 (CBF) $w: \square \forall x A \Rightarrow w: \forall x \square A$

$$
\begin{aligned}
& \text { Proof }
\end{aligned}
$$

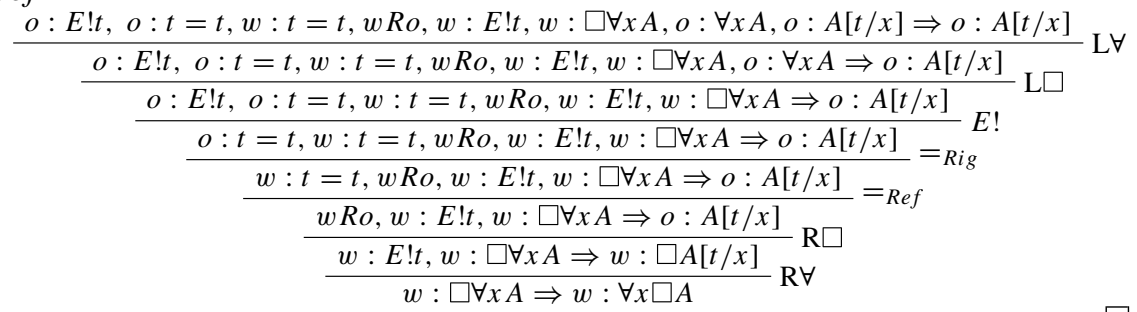




$$
\frac{o: E ! t, o R w, w: E ! t, \Gamma \Rightarrow \Delta}{o R w, w: E ! t, \Gamma \Rightarrow \Delta} E ! c \quad \frac{o: E ! t, w R o, w: E ! t, \Gamma \Rightarrow \Delta}{w R o, w: E ! t, \Gamma \Rightarrow \Delta} E ! e
$$

Fig. 9 Contracting and Expanding $E$ ! rules

Lemma 6.15 (BF) $w: \forall x \square A \Rightarrow w: \square \forall x A$

$$
\begin{aligned}
& \text { Proof }
\end{aligned}
$$

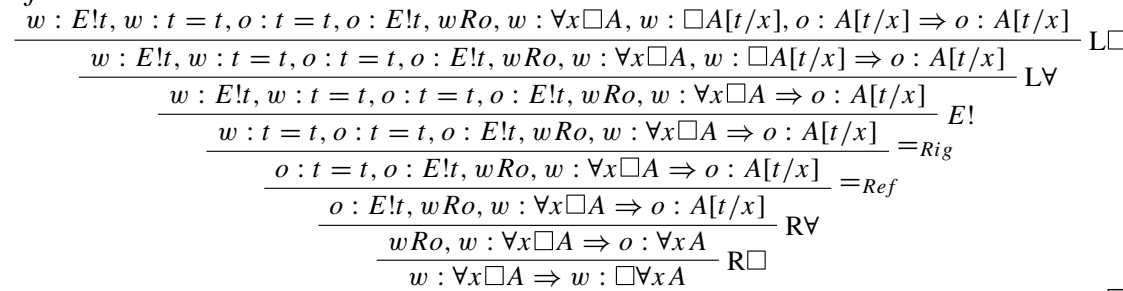

Such a situation does not occur in the positive case, since there is no close connection between the existence and identity predicates. This is, to the best of our knowledge, the first quantified modal logic based on the negative free logic, and it is interesting to note that in $G 3 K_{\text {inf }}$ the Barcan formulas come about as the result of the rigidity of names. However, it should be clear that it is really the invariance of the $E$ ! predicate that establishes said formulas. So, on any variant we can simply add a rule for invariance of $E$ ! and obtain the same results.

Moreover, while adding such a rule will yield both the Barcan formula and its converse, we can make the result more fine-grained by adding two separate rules:

It is straightforward to show that these will allow the derivation of the Barcan formula and the Converse Barcan formula, respectively.

$$
\frac{w: E ! t, o: E ! t, w R o, w: \forall x \square A, w: \square A[t / x], o: A[t / x] \Rightarrow o: A[t / x]}{\frac{w: E ! t, o: E ! t, w R o, w: \forall x \square A, w: \square A[t / x] \Rightarrow o: A[t / x]}{\frac{w: E ! t, o: E ! t, w R o, w: \forall x \square A \Rightarrow o: A[t / x]}{o: E ! t, w R o, w: \forall x \square A \Rightarrow o: A[t / x]}} \mathrm{R} \forall \forall} \mathrm{R} \forall
$$

Parallel for CBF. The connection with the rules for the contracting and expanding domains is unmistakable, and so this serves to further illustrate that in free logic $E$ ! serves as the domain marker.

\section{Concluding Remarks}

In this paper we have established and systematized a range of sequent calculi for free logics. Utilizing the similarity between modal operators and quantifiers, but working from the former to the latter, we formulated the quantifier rules for free logic in a way 
that treats $E$ ! as a relational atom. This proved to carry several benefits - in addition to simplification of the meta-theoretical and structural proofs, it also allowed us to construct systems for free logic where the rules do not rely on implicit implication and conjunction for the rules $\mathrm{L} \forall$ and $\mathrm{R} \exists$, respectively.

Moreover, by generalizing the semantic restrictions in a way that is still adequate for the sequent calculus rules, we have obtained a system of semantics that enables us to deal with a multitude of different systems of free logic. These approaches tend to be mutually incompatible as well as difficult to extricate from their philosophical motivations. The approach taken in this paper allows, on the other hand, for a prooftheoretic examination of free logics while avoiding such pitfalls.

The possibilities of application of our results have not been exhausted in this paper - specifically, even more unification is possible. In the introduction to this paper we mentioned and described briefly the core idea of neutral free logics. It is on our future research agenda to see if one (or more versions) of this family of free logic can be integrated in our new framework. Having system(s) for a neutral free logic seems timely and interesting also when viewed from a different perspective: hyperintensional logic, e.g. [13]. In [13] the hyperintensional logic is construed as an extension of intuitionistic logic with the constant domain axiom. Viewed from this angle it is also natural to construct free logics (including neutral ones) within the ever growing new field of hyperintensional logics.

Moreover, [14] demonstrates the usefulness of the single-premise approach to quantification in obtaining the Craig's interpolation theorem. We also plan to extend those results to the systems developed here. And finally, we can also see a further avenue for unification in bringing systems with intutionistic base into our framework.

Funding Information Open access funding provided by University of Helsinki including Helsinki University Central Hospital. This paper was written with support of the Academy of Finland research project no. 1308664. This paper was written with support of the Deutsche Forschungsgemeinschaft (DFG) research project no. 390218268

Open Access This article is licensed under a Creative Commons Attribution 4.0 International License, which permits use, sharing, adaptation, distribution and reproduction in any medium or format, as long as you give appropriate credit to the original author(s) and the source, provide a link to the Creative Commons licence, and indicate if changes were made. The images or other third party material in this article are included in the article's Creative Commons licence, unless indicated otherwise in a credit line to the material. If material is not included in the article's Creative Commons licence and your intended use is not permitted by statutory regulation or exceeds the permitted use, you will need to obtain permission directly from the copyright holder. To view a copy of this licence, visit http://creativecommonshorg/licenses/by/4.0/.

\section{Appendix A: Structural properties}

Lemma 2.4 ( $\alpha$-conversion) A derivation (where $\vdash_{n}$ denotes derivability with height bounded by $n$ ) of $\vdash_{n} \Gamma \Rightarrow \Delta$ can be converted into a derivation of $\vdash_{n} \Gamma^{\prime} \Rightarrow \Delta^{\prime}$, where $\Gamma^{\prime}$ and $\Delta^{\prime}$ differ from $\Gamma$ and $\Delta$, respectively, only in namings of bound variables.

Proof By induction on the height of a derivation. 
If $\Gamma \Rightarrow \Delta$ is an initial sequent, then so is $\Gamma^{\prime} \Rightarrow \Delta^{\prime}$. If the last rule is any of the propositional or identity rules, the namings remain unchanged from the premis(es). If the last rule applied is $\mathrm{R} \forall$, then it is derived from some sequent $E ! t, \Gamma \Rightarrow \Delta, A[t / x]$. By the inductive hypothesis $E ! t, \Gamma^{\prime} \Rightarrow \Delta^{\prime}, A[y / x][t / y]$ is likewise derivable, so by applying $\mathrm{R} \forall$ we obtain $\Gamma^{\prime} \Rightarrow \Delta^{\prime}, \forall y A[y / x]$. Similar for other quantifier rules and simple for the rule $E$ !.

Lemma 2.5 (Substitution) If $\vdash_{n} \Gamma \Rightarrow \Delta$ is derivable in $G 3_{p f}\left(G 3_{n f}\right)$, then $\vdash_{n}$ $\Gamma[t / s] \Rightarrow \Delta[t / s]$ is derivable.

Proof By induction on the height of the derivation. If $\Gamma \Rightarrow \Delta$ is an initial sequent, then so is $\Gamma[t / s] \Rightarrow \Delta[t / s]$.

Propositional rules do not alter the free and bound individual variables between their premis(es) and conclusion. The rules $=_{\text {Ref }}$ and $E$ ! in $G 3_{n f}$ also do not alter the free and bound individual variables between their premise and conclusion, and the step is straightforward for $=_{R e f}$ in $G 3_{p f}$. The rule $={ }_{R e p l}$ does not alter the free and bound individual variables.

If the last rule applied is $\mathrm{R} \forall$ and $t$ is an eigenvariable of that application of the rule (otherwise we skip the first application of the inductive hypothesis), the premise of the application of the rule is some $E ! t, \Gamma \Rightarrow \Delta, A[t / x]$. We use the inductive hypothesis to replace it with some $d$ that has not so far occurred anywhere above that application of the rule to obtain $E ! d, \Gamma \Rightarrow \Delta, A[t / x][d / t]$ (we know by eigenvariable condition that $t$ does not occur in $\Gamma$ or $\Delta$ ), which is the same as $E ! d, \Gamma \Rightarrow \Delta, A[d / x]$. Using the inductive hypothesis again we get $E ! d, \Gamma[t / s] \Rightarrow \Delta[t / s], A[d / x][t / s]$, which is the same as $E ! d, \Gamma[t / s] \Rightarrow \Delta[t / s], A[t / s][d / x]$. Now we apply $\mathrm{R} \forall$ to obtain $\Gamma[t / s] \Rightarrow \Delta[t / s], \forall x A[t / s]$. Similar for other quantifier rules.

Lemma 2.6 (Axiom generalization) Any sequent of the form $A, \Gamma \Rightarrow \Delta, A$ is derivable in $G 3_{p f}$ and $G 3_{n f}$.

Proof By induction on the weight of $A$. Since the two systems do not differ in their language, the proofs proceed the same. The interesting cases here are for quantified formulas:

$$
\begin{gathered}
\frac{\vdots}{A[t / x], E ! t, \forall x A, \Gamma \Rightarrow \Delta, A[t / x]} \\
\frac{E ! t, \forall x A, \Gamma \Rightarrow \Delta, A[t / x]}{\forall x A, \Gamma \Rightarrow \Delta, \forall x A} \mathrm{R} \forall \\
\frac{{ }^{E ! t, A[t / x], \Gamma \Rightarrow \Delta, \exists x A, A[t / x]}}{\frac{E ! t, A[t / x], \Gamma \Rightarrow \Delta, \exists x A}{\exists} \mathrm{L} \exists} \mathrm{R} \exists
\end{gathered}
$$


Lemma 2.7 (Weakening) Weakening is height-preserving admissible in G3 $3_{p f}$ and $G 3_{n f}$ :

i) If $\vdash_{n} \Gamma \Rightarrow \Delta$ then $\vdash_{n} C, \Gamma \Rightarrow \Delta$.

ii) If $\vdash_{n} \Gamma \Rightarrow \Delta$ then $\vdash_{n} \Gamma \Rightarrow \Delta, C$.

Proof Routine by induction on the height of the derivation, using Lemma 2.5 when necessary.

Lemma 2.8 (Invertibility) All the rules of $G 3_{p f}$ and $G 3_{n f}$ are height-preserving invertible.

Proof Straightforward for propositional rules, follows from Lemma 2.7 for all other rules except $\mathrm{R} \forall$ and $\mathrm{L} \exists$. So what remains to be shown is:

i) If $\vdash_{n} \Gamma \Rightarrow \Delta, \forall x A$, then $\vdash_{n} E ! t, \Gamma \Rightarrow \Delta, A[t / x]$, and

ii) if $\vdash_{n} \exists x A, \Gamma \Rightarrow \Delta$, then $\vdash_{n} E ! t, A[t / x], \Gamma \Rightarrow \Delta$.

We demonstrate (i) by induction on the height of the derivation, $n$.

If $n=0$, then $\Gamma \Rightarrow \Delta, \forall x A$ is an initial sequent, but then so is $E ! t, \Gamma \Rightarrow$ $\Delta, A[t / x]$.

If $n>0$, then if $\forall x A$ is not principal in the last step, $\Gamma \Rightarrow \Delta, \forall x A$ follows from some sequents $E ! t, \Gamma^{\prime} \Rightarrow \Delta^{\prime}, \forall x A\left(E ! t, \Gamma^{\prime \prime} \Rightarrow \Delta^{\prime \prime}, \forall x A\right)$ with height $\leq n-1$. Applying the Lemma 2.5 if $t$ is an eigenvariable of the application of the rule and then the inductive hypothesis, we obtain $E ! t, \Gamma^{\prime} \Rightarrow \Delta^{\prime}, A[t / x]\left(E ! t, \Gamma^{\prime \prime} \Rightarrow \Delta^{\prime \prime}, A[t / x]\right)$, and then applying the rule again we get $E ! t, \Gamma \Rightarrow \Delta, A[t / x]$.

If on the other hand $\forall x A$ is principal, then the upper sequent of the last application of the rule is already of the required form and with height $\leq n$.

Parallel for (ii).

Lemma 2.9 (Contraction) Contraction is height-preserving admissible in G3 pf and $G 3_{n f}$ :

i) If $\vdash_{n} C, C, \Gamma \Rightarrow \Delta$ then $\vdash_{n} C, \Gamma \Rightarrow \Delta$.

ii) If $\vdash_{n} \Gamma \Rightarrow \Delta, C, C$ then $\vdash_{n} \Gamma \Rightarrow \Delta, C$.

Proof Simultaneous for (i) and (ii) by induction on the height of the derivation. The interesting part is when the formula $C$ is principal in $\mathrm{R} \forall$ or $\mathrm{L} \exists$.

So, assume that $C$ is $\forall x A$ and principal in $\mathrm{R} \forall$ in the last step. Then the last step of the derivation is $\Gamma \Rightarrow \Delta, \forall x A, \forall x A$, derived by $\mathrm{R} \forall$ from $E ! t, \Gamma \Rightarrow \Delta, \forall x A, A[t / x]$. Applying the Lemma 2.8 to that sequent we get $E ! t, E ! t, \Gamma \Rightarrow \Delta, A[t / x], A[t / x]$ with the same height of $\leq n-1$. We then apply the inductive hypothesis to obtain $E ! t, \Gamma \Rightarrow \Delta, A[t / x]$ and then $\mathrm{R} \forall$ to finally obtain $\Gamma \Rightarrow \Delta, \forall x A$. Similar for $\mathrm{L} \exists$. 
Theorem 2.10 Cut is admissible in $G 3_{p f}$ and $G 3_{n f}$.

Proof By induction on the weight of a formula and subinduction on the sum of heights of the two upper sequents of a cut. Standard for propositional rules, identity rules in $G 3_{p f}$ and the rule $=_{R e p l}$ in $G 3_{n f}$. We check the remaining cases.

When the cut formula is principal in the rule $E$ ! the cut has the following form:

$$
\frac{\Gamma_{1} \Rightarrow \Delta_{1}, P[t] \quad \frac{E ! t, P[t], \Gamma_{2} \Rightarrow \Delta_{2}}{P[t], \Gamma_{2} \Rightarrow \Delta_{2}} \mathrm{Cut}}{\Gamma_{1}, \Gamma_{2} \Rightarrow \Delta_{1}, \Delta_{2}}
$$

If $\Gamma_{1} \Rightarrow \Delta_{1}, P[t]$ is an initial sequent we distinguish two cases. First, if $\Gamma_{1} \Rightarrow$ $\Delta_{1}, P[t]$ is of the form $P[t], \Gamma_{1}^{\prime} \Rightarrow \Delta_{1}, P[t]$ (i.e. if $\Gamma_{1}$ contains $P[t]$ ), then the bottom sequent of the cut is of the form $P[t], \Gamma_{1}^{\prime}, \Gamma_{2} \Rightarrow \Delta_{1}, \Delta_{2}$ and can be obtained from $P[t], \Gamma_{2} \Rightarrow \Delta_{2}$ using Lemma 2.7. Second, if $\Gamma_{1}$ does not contain $P[t]$, then $\Gamma_{1} \Rightarrow \Delta_{1}$ is likewise an initial sequent.

If $\Gamma_{1} \Rightarrow \Delta_{1}, P[t]$ is not initial then the derivation has the following form (since an atomic formula cannot be principal on the right):

$$
\frac{\Gamma_{1}^{\prime} \Rightarrow \Delta_{1}^{\prime}, P[t] \quad\left[\Gamma_{1}^{\prime \prime} \Rightarrow \Delta_{1}^{\prime \prime}, P[t]\right]}{\frac{\Gamma_{1} \Rightarrow \Delta_{1}, P[t]}{\Gamma_{1}, \Gamma_{2} \Rightarrow \Delta_{1}, \Delta_{2}} \quad \frac{E ! t, P[t], \Gamma_{2} \Rightarrow \Delta_{2}}{P[t], \Gamma_{2} \Rightarrow \Delta_{2}} \mathrm{Cut}} E !
$$

This is transformed into:

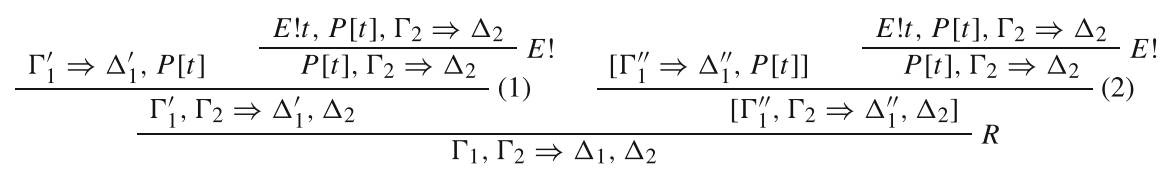

Here cuts numbered (1) and (2) are of lesser height. Similar for the rule $={ }_{\text {Ref }}$ of $G 3_{n f}$.

When the cut formulas are quantified the interesting case is when they are principal in both upper sequents. In the case of $\forall$, the cut is then of the form:

$$
\frac{E ! s, \Gamma_{1} \Rightarrow \Delta_{1}, A[s / x]}{\frac{\Gamma_{1} \Rightarrow \Delta_{1}, \forall x A}{R} \forall \quad \frac{A[t / x], E ! t, \forall x A, \Gamma_{2} \Rightarrow \Delta_{2}}{E ! t, \forall x A, \Gamma_{2} \Rightarrow \Delta_{2}} \mathrm{Cut}} \mathrm{L} \forall
$$

This is transformed into:

$$
\frac{\frac{E ! s, \Gamma_{1} \Rightarrow \Delta_{1}, A[s / x]}{E ! t, \Gamma_{1} \Rightarrow \Delta_{1}, A[t / x]} \operatorname{Lem} 2.5 \quad \frac{\Gamma_{1} \Rightarrow \Delta_{1}, \forall x A \quad A[t / x], E ! t, \forall x A, \Gamma_{2} \Rightarrow \Delta_{2}}{A[t / x], E ! t, \Gamma_{1}, \Gamma_{2} \Rightarrow \Delta_{1}, \Delta_{2}}}{\frac{E ! t, E ! t, \Gamma_{1}, \Gamma_{1}, \Gamma_{2} \Rightarrow \Delta_{1}, \Delta_{1}, \Delta_{2}}{E ! t, \Gamma_{1}, \Gamma_{2} \Rightarrow \Delta_{1}, \Delta_{2}} \text { Lemma } 2.9}
$$


Here the cut numbered (1) is of lesser weight and (2) are of lesser height. Similar for $\exists$.

\section{Appendix B: Adequacy of axioms}

Theorem 2.16 All of the PFL axioms A1-A6 are derivable in G3 $3_{p f}$.

Proof A1: $\forall x(A \rightarrow B) \rightarrow(\forall x A \rightarrow \forall x B)$

Lemma 2.17

$$
\begin{gathered}
\frac{A[t / x] \rightarrow B[t / x], A[t / x], E ! t, \forall x(A \rightarrow B), \forall x A \Rightarrow B[t / x]}{\frac{A[t / x], E ! t, \forall x(A \rightarrow B), \forall x A \Rightarrow B[t / x]}{E ! t, \forall x(A \rightarrow B), \forall x A \Rightarrow B[t / x]}} \mathrm{L} \forall \\
\frac{\forall x(A \rightarrow B), \forall x A \Rightarrow \forall x B}{\forall x(A \rightarrow B) \Rightarrow \forall x A \rightarrow \forall x B} \mathrm{R} \rightarrow
\end{gathered}
$$

A2: $\quad A \rightarrow \forall x A$

$$
\frac{\frac{\text { Lemma } 2.6}{E ! t, A \Rightarrow A[t / x]}}{A \Rightarrow \forall x A} \mathrm{R} \forall
$$

A3: $\forall x A \rightarrow(E ! t \rightarrow A[t / x])$

$$
\frac{A[t / x], \forall x A, E ! t \Rightarrow A[t / x]}{\forall x A, E ! t \Rightarrow A[t / x]} \mathrm{L} \forall
$$

A4: $\forall x E ! x$

$$
\frac{E ! t \Rightarrow E ! t}{\Rightarrow \forall x E ! x} \mathrm{R} \forall
$$

$\mathrm{A} 5: \quad s=t \rightarrow(A \rightarrow A[t / / s])$

$$
\frac{\frac{\text { Lemma } 2.18}{s=t, A \Rightarrow A[t / / s]}}{s=t \Rightarrow A \rightarrow A[t / / s]} \mathrm{R} \rightarrow
$$

A6: $t=t$

$$
\frac{t=t \Rightarrow t=t}{\Rightarrow t=t}={ }_{R e f}
$$


Theorem 2.17 All of the NFL axioms Al-A7 are derivable in $G 3_{n f}$.

Proof Since most of the axioms are the same in both systems, it only remains to show that the different version of A6, and the new axiom A7, are derivable.

A6: $\forall x(x=x)$

$$
\begin{gathered}
\frac{E ! t, t=t \Rightarrow t=t}{E ! t \Rightarrow t=t}=R e f \\
\frac{R}{\Rightarrow \forall x(x=x)} \mathrm{R} \forall
\end{gathered}
$$

A7: $P t_{1}, \ldots, t_{n} \rightarrow E ! t_{i}, 1 \leq i \leq n$

$$
\frac{E ! t_{i}, P t_{1}, \ldots, t_{n} \Rightarrow E ! t_{i}}{P t_{1}, \ldots, t_{n} \Rightarrow E ! t_{i}} E !
$$

\section{References}

1. Antonelli, G. (2000). Proto-Semantics for positive free logic. Journal of Philosophical Logic, 29, 277-294.

2. Bencivenga, E. (2002). Free logics. In Gabbay, D., \& Guenthner, F. (Eds.) Handbook of philosophical logic, (Vol. 5 pp. 147-196). Dordrecht: Springer.

3. Berry, G.D.W. (1941). On Quine's axioms of quantification. The Journal of Symbolic Logic, 6, $23-27$.

4. Feferman, S. (1995). Definedness. Erkenntnis, 43, 295-320.

5. Garson, J. (1991). Applications of free logic to quantified intensional logic. In Lambert, K. (Ed.) Philosophical applications of free logic, (Vol. 5 pp. 111-142). New York: Oxford University Press.

6. Gratzl, N. (2010). A sequent calculus for a negative free logic. Studia Logica, 96, 331348 .

7. Hailperin, T. (1953). Quantification theory and empty individual domains. The Journal of Symbolic Logic, 18, 197-200.

8. Hintikka, J. (1959). Existential presuppositions and existential commitments. The Journal of Philosophy, 56, 125-137.

9. Lambert, K. (1967). Free logic and the concept of existence. Notre Dame Journal of Formal Logic, 8, $133-144$.

10. Lambert, K. (1997). Free logics: their foundations, character, and some applications thereof. Sankt Augustin: Academia Verlag.

11. Lambert, K. (2001). Free logics. In Goble, L. (Ed.) The Blackwell guide to philosophical logic (pp. 258-279). Malden, Oxford, Carlton, Berlin: Blackwell Publishers.

12. Lehmann, S. (2002). More free logics. In Gabbay, D., \& Guenthner, F. (Eds.) Handbook of philosophical logic, (Vol. 5 pp. 197-259). Dordrecht: Springer.

13. Leitgeb, H. (2019). HYPE: A system of hyperintensional logic (with an application to semantic paradoxes). Journal of Philosophical Logic, 48, 305-405.

14. Maffezioli, P., \& Orlandelli, E. (2019). Full cut elimination and interpolation for intuitionistic logic with existence predicate. Bulletin of the Section of Logic, 48(2), 137-158.

15. Morscher, E., \& Hieke, A. (2013). New essays in free logic: In honour of Karel Lambert. Dordrecht: Springer.

16. Negri, S. (2005). Proof analysis in modal logic. Journal of Philosophical Logic, 34, 507-544.

17. Negri, S., \& Orlandelli, E. (2019). Proof theory for quantified monotone modal logics. Logic Journal of the IGPL, 27, 478-506. 
18. Negri, S., \& von Plato, J. (1998). Cut elimination in the presence of axioms. The Bulletin of Symbolic Logic, 4, 418-435.

19. Negri, S., \& von Plato, J. (2001). Structural proof theory. Cambridge: Cambridge University Press.

20. Negri, S., \& von Plato, J. (2011). Proof analysis: A contribution to Hilbert's last problem. Cambridge: Cambridge University Press.

21. Nolt, J. (2018). Free Logic. In Zalta, E.N. (Ed.) The Stanford Encyclopedia of Philosophy (Fall 2018 Edition) https://plato.stanford.edu/archives/fall2018/entries/logic-free/. Accessed 13 December 2019.

22. Pavlović, E., \& Gratzl, N. (2019). Proof-Theoretic analysis of the Quantified argument calculus. The Review of Symbolic Logic, 12(4), 607-636.

23. Quine, W.V. (1954). Quantification and the empty domain. The Journal of Symbolic Logic, 19, 177179.

24. Troelstra, A., \& Schwichtenberg, H. (2000). Basic proof theory. Cambridge: Cambridge University Press.

Publisher's Note Springer Nature remains neutral with regard to jurisdictional claims in published maps and institutional affiliations.

\section{Affiliations}

\section{Edi Pavlović ${ }^{1}$ (D) - Norbert Gratzl ${ }^{2}$}

Norbert Gratzl

N.Gratzl@1mu.de

1 Department of Philosophy, History and Art Studies, University of Helsinki, P.O. Box 24, FI-00014, Helsinki, Finland

2 Fakultät für Philosophie, Wissenschaftstheorie und Religionswissenschaft, Munich Center for Mathematical Philosophy (MCMP), Ludwig-Maximilians Universität München, Geschwister Scholl Platz 1, 80539, München, Germany 